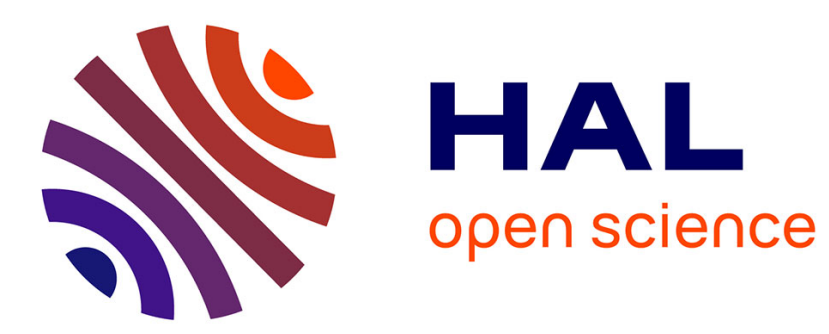

\title{
Optimal Design of a 6-dof Parallel Measurement Mechanism Integrated in a 3-dof Parallel Machine-Tool
}

David Corbel, Olivier Company, François Pierrot

\section{To cite this version:}

David Corbel, Olivier Company, François Pierrot. Optimal Design of a 6-dof Parallel Measurement Mechanism Integrated in a 3-dof Parallel Machine-Tool. IROS'08: International Conference on Intelligent RObots and Systems, Sep 2008, Nice, France. pp.1970-1976, 10.1109/IROS.2008.4650930 . lirmm-00304811v2

\section{HAL Id: lirmm-00304811 https://hal-lirmm.ccsd.cnrs.fr/lirmm-00304811v2}

Submitted on 23 Feb 2009

HAL is a multi-disciplinary open access archive for the deposit and dissemination of scientific research documents, whether they are published or not. The documents may come from teaching and research institutions in France or abroad, or from public or private research centers.
L'archive ouverte pluridisciplinaire HAL, est destinée au dépôt et à la diffusion de documents scientifiques de niveau recherche, publiés ou non, émanant des établissements d'enseignement et de recherche français ou étrangers, des laboratoires publics ou privés. 


\title{
Optimal Design of a 6-dof Parallel Measurement Mechanism Integrated in a 3-dof Parallel Machine-Tool
}

\author{
David Corbel, Olivier Company and François Pierrot \\ Univ. Montpellier 2, CNRS, LIRMM, 161 rue Ada \\ 34392 Montpellier, France \\ Email: david.corbel@lirmm.fr \\ Phone: +33/0 467418585 \\ Fax: $+33 / 0467418500$
}

\begin{abstract}
This paper presents the design and the optimization of a parallel machine-tool composed of (i) an 3-dof actuated parallel mechanism (a linear Delta) and (ii) a 6-dof measuring parallel mechanism (a Gough platform). The interest to use a measuring device independent of the actuation device is shown and the modeling of both devices used for the optimization is explained. Then, the optimization of both mechanisms is presented. The optimization of the actuated mechanism is performed to have an homogeneous behavior in velocity while the goal of the measuring device optimization is to obtain the best resolution for the measuring system evaluated at the tool level.
\end{abstract}

\section{INTRODUCTION}

Machine-tool (MT) builders are always looking for better performances in terms of accuracy, speed, productivity and stiffness. Naturally, MT designers took their inspiration from recent advances in robot kinematic architectures, in particular Parallel Kinematics Machines (PKMs) [1]. PKMs have nowadays shown their efficiency in some robotic domains and commercial robots are widely available today ${ }^{1}$.

Among the transfers from well known robotic PKMs to the machine tool industry, one can cite:

- The hexapods, where six variable length struts link a moving traveling plate to a base. One of the first built PKMs belonging to this family was proposed by Gough [2], and the first MT inspired by this architecture was the Variax [3]. Until today, a lot of industrial machines are built such as Octahedral Hexapod by Ingersoll, the P1000/P2000/P3000 Hexapods by PRSCO, and the SEYANKA hexapod milling machine by Tekniker.

- The Delta kinematics invented by prof Clavel [4] is lower mobility PKM (displacements of the traveling plate are restricted to three translations). It is a light weight structure having intrinsically high dynamic performances. Robots based on this architecture are widely available (see, for example FlexPicker by ABB). MTs based on the Delta robot principle were also designed such as UraneSX [5] that can reach up to $4 g$ ( $g=$ $9,81 \mathrm{~m} . \mathrm{s}^{-2}$ ) in its workspace or the Quickstep by Krause \& Mauser [6].

Whatever the architecture is, geometrical calibration is required to get the best accuracy performances of the MTs. The

\footnotetext{
${ }^{1}$ http://www.parallemic.org/WhosWho/CompPKM.html
}

calibration tries to identify model parameters that enhance machine accuracy [7]. Once these parameters are identified, the model runs "open loop", i. e. the machine behavior is expected to be the one that has been modeled and identified whatever the deformations (elastic deformations, thermal expansion, etc.) in machine components are. For Cartesian serial MTs, the identification can be done axis per axis. Parameter identification can be very accurate as the problem is decoupled. Identifying PKM parameters, according to this principle, is not possible as all axes are, generally, coupled in the model. A full calibration of the model must be done, but it always ends in a compromise between the number of parameters and the numerical stability.

Moreover for MTs based on parallel architectures, geometrical calibration is not sufficient. To benefit to the high dynamics of the parallel architecture, the use of light elements is necessary and therefore the stiffness of the machine can be low [8]. This weak stiffness of parallel mechanisms is a drawback when they are used in MTs. One solution consists to add redundancy in the architecture to improve the mechanism stiffness [9]. Another solution consists in modeling the deformations with an elastic model of the structure [10] [11] [12]. This solution is interesting to compensate the gravity effects or when the stresses on the tool are known but it is seldom the case.

The basic problem in machining is to impose accurate tool positioning regarding the part to be machined. The best way to deal with accuracy is to be always able to know the tool position accurately, i. e. with a quality as close as possible to a metrological one. This can be done by a device which measures, continuously, the position of the tool. But this measuring device must be independent of the actuation device to avoid the perturbations on the measurement due to the stresses applied on the tool. Two solutions can be considered: a non-contact full pose measurement system or a mechanical measuring device.

If we consider the first solution, vision systems or laser based systems can be used. But, there is still ongoing research on the vision measuring system and, even if algorithms are available, they are not able today to guarantee the requested resolution on the whole workspace of the machine [13]. Moreover, the refreshment rate is not high enough for the control loop, but it is still a promising way of research for 
the future. Concerning laser based systems, they are too expensive and the non-contact laser measuring systems like laser tracker cannot measure orientation.

The second solution is to build a mechanical structure, with metrological considerations, which is able to give information to compute the actual tool pose [14]. This mechanical structure needs to have a good resolution and a good accuracy and it must not transmit any stresses. Hence, the design of this mechanism must be optimized to obtain the best accuracy as possible. But dimensional synthesis of a mechanism from error analysis is a complex problem [15] [16] [17] [18]. Our approach is based on the error analysis presented in [19]. In fact, from the given resolution of the metrological mechanism encoder, we look for the best dimensions of this mechanism to have the best measuring resolution evaluated at the tool level.

To prove the feasibility and the efficiency of this concept, a PKM MT architecture (Delta) must be firstly selected and then a measuring architecture (Gough platform) is defined. Justification, description and modeling are given in section II. As it is well known that behavior of PKMs depends strongly on their design parameters, an optimization for both mechanisms is done in section III and IV. Conclusion and future works are given in section $\mathrm{V}$.

\section{MACHINE DESCRIPTION}

\section{A. Selection of the architectures}

1) Actuation architecture: Basic machining operations (grooving, drilling, contouring) require three translational degrees of freedom (dof). We must select an architecture that provides these dof while constraining the three rotational dof to a constant value. Several hybrid mechanisms or PKMs are able to provide these dof [20]. Among them, one can cite the Tsai mechanism [21], the Star mechanism [22], and the Speed-R-Man mechanism [23].

But, the architecture that guarantees intrinsically the highest dynamic performances is the Delta mechanism. For MTs, linear actuation is preferred to make it as mechanically stiff as possible. So the traveling plate will be actuated by a linear Delta, as in the UraneSX MT. The Delta architecture theoretically imposes a constant orientation of the traveling plate and allows controlling three translations. But due to the manufacturing and assembly errors, and the elastic deformations of machine elements, it is not possible to guarantee that no parasitic rotation of the platform occurs. These rotations impairs machine accuracy because of the varying lever arm (depends on tool length and position of tool cutting edge) between the tool extremity and the moving platform. The consequence is that the measuring device to be integrated to the machine must be able to measure the $x, y, z$ position of the tool, but also its parasitic rotation to forecast the imperfect shape of the machined part.

2) Measurement architecture: As mentioned before, a full pose measurement system is required. Non contact system based on vision are, today, not accurate enough and cannot guarantee a fast refresh rate compatible with control loops.
Concerning the existing non contact measuring system based on laser (like laser tracker), they are too expensive and cannot measure directly the orientation of the measured object. We propose here to use a mechanical measuring system. A strong constraint on this measuring system is that it will be attached on one side to the fixed base of the machine and on the other side on the moving traveling plate. The problem is that the traveling plate is expected to move in machine workspace with a high acceleration capability. The measuring system must not reduce this acceleration capability. The consequence is that it must be light weight. Then, this mechanical measuring device must not transmit any stresses to insure a good accuracy. The kinematics of the system must take account of it. Moreover collision considerations with the actuation architecture must be taken into consideration for avoiding any restriction of machine workspace.

To measure the position and the orientation of a solid, six measurements are necessary. These measurements can be distance measurements or angle measurements. To respect the Abbe's principle and to avoid the angular error amplifications due to lever arm, distance measurements are chosen. From there, the choice of parallel architecture is natural because measure an orientation from distance measurement is impossible with serial architecture. The most common and the simplest 6-dof parallel architecture is the hexapod. Many arrangements of hexapod exist such as the system MAST (Multi-Axis Simulation (or Shake) Table), the Gough platform, the Stewart platform ${ }^{2}$. The system MAST is very interesting because the strut adjustments would be simple and interpretable for small variations. But in our case we need quite big variations of the leg lengths to insure a good MT workspace. Then, the system MAST has a singularity which is close to the MT workspace ; that is decreased the accuracy of the measuring device. Moreover, this system takes up too much space.

An other type of hexapod is best suited to our machine: the Gough platform. This mechanism is very compact and can be placed behind the Delta mechanism away from the working area (see Fig. 1). Its realization is very simple because six take-down variable length struts are used. These struts link the base to the traveling plate simply with spheres and magnets; this facilitate the calibration of such a system as we will see below.

\section{B. Modeling of the linear Delta mechanism}

Several arrangements of the Delta exists [4] [24] [25]. In our case, a simple robot Delta with prismatic actuators is chosen (Fig. 1). The motor axis are placed at $120^{\circ}$ to each other. The only useful parameters of the Delta mechanism are:

- $\Delta R$, the difference between the radius of the base, $R_{B}$ and the radius of the traveling plate, $R_{T P}$

- $L$, the length of the arms

- $d$, the distance between points $C_{i}$ and $C_{i j}$ (and points $D_{i}$ and $D_{i j}$ ).

${ }^{2}$ http://www.parallemic.org/Reviews/Review007.html 


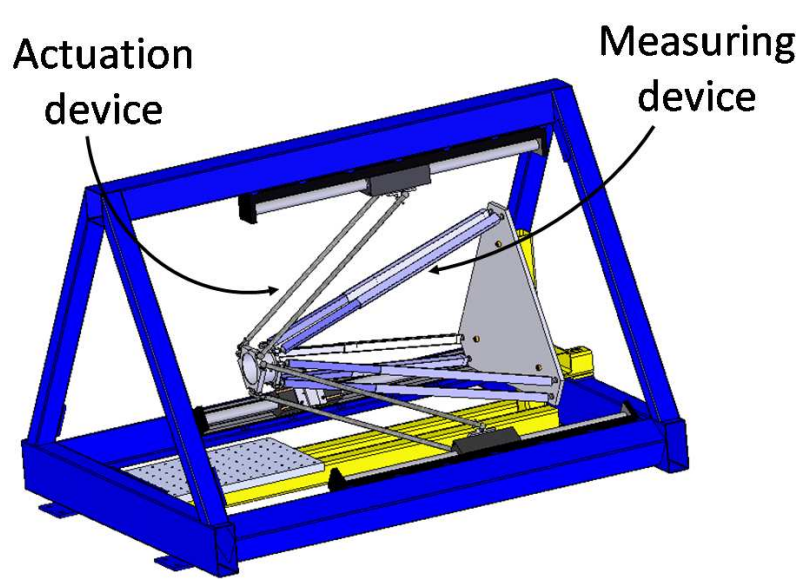

(a) $3 \mathrm{D}$ view

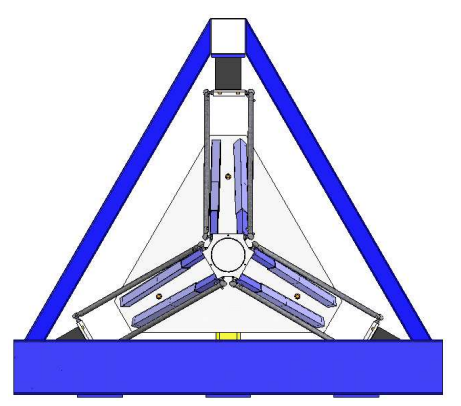

(b) Front view

Fig. 1. Measuring device location

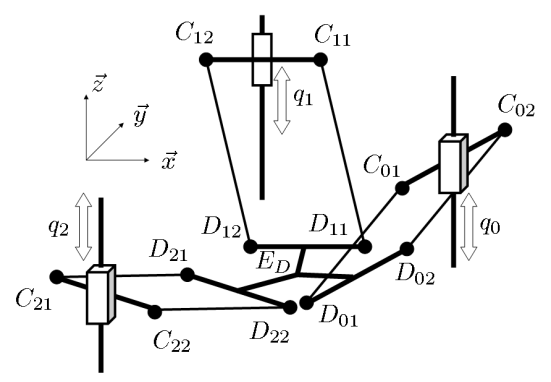

(a) $3 \mathrm{D}$ view

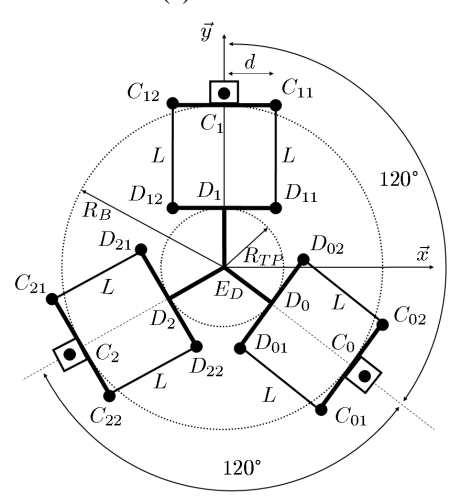

(b) Top view
Figure 2 shows the geometrical parameters of the Delta mechanism. The coordinates of the traveling plate center $E_{D}$ are $x_{D}, y_{D}, z_{D}$ and the active-joint variables are $q_{0}, q_{1}, q_{2}$. The optimization of the Delta mechanism presented in this paper is based on the study of the condition number of the Jacobian matrix $\boldsymbol{J}_{D}$ which links operational speed $\dot{\boldsymbol{x}}_{D}$ to joints velocities $\dot{\boldsymbol{q}}_{D}$ :

$$
\dot{\boldsymbol{x}}_{D}=\boldsymbol{J}_{D} \dot{\boldsymbol{q}}_{D}
$$

The matrix $\boldsymbol{J}_{D}$ is given by:

$$
\boldsymbol{J}_{D}=\boldsymbol{J}_{x}^{-1} \boldsymbol{J}_{q}
$$

where

$$
\begin{gathered}
\boldsymbol{J}_{x}=\left[\begin{array}{ccc}
x_{D}-\frac{\Delta R \sqrt{3}}{2} & y_{D}+\frac{\Delta R}{2} & z_{D}-q_{0} \\
x_{D} & y_{D}-\Delta R & z_{D}-q_{1} \\
x_{D}+\frac{\Delta R \sqrt{3}}{2} & y_{D}+\frac{\Delta R}{2} & z_{D}-q_{2}
\end{array}\right] \\
\boldsymbol{J}_{q}=\left[\begin{array}{ccc}
z_{D}-q_{0} & 0 & 0 \\
0 & z_{D}-q_{1} & 0 \\
0 & 0 & z_{D}-q_{2}
\end{array}\right]
\end{gathered}
$$

\section{Modeling of the Gough platform}

Figure 3 presents the parameters of the Gough platform. Points $A_{i}\left(B_{i}\right)$ which represents the centers of the spherical joints on the base (on the traveling plate) are placed on a circle of radius $r_{b}\left(r_{T P}\right)$. Then, three lines passing by the base center $O$ and the traveling plate center $T_{H}$ and separated by an angle $\alpha_{0}$ are defined. Points $A_{i}\left(B_{i}\right)$ are then located symmetrically to these lines, two by two, with an angle of $\alpha_{b}\left(\alpha_{T P}\right)$.

The measured-joint variables are defined by the hexapod leg lengths noted $l_{i}(i \in[1,6])$. The pose $\boldsymbol{x}_{H}$ of the platform is defined by the coordinates $x_{H}, y_{H}, z_{H}$ of the traveling plate center $E_{H}$ in the base frame $\mathcal{R}_{b}$ together with 3 angles $\psi_{H}, \theta_{H}, \phi_{H}$ that allows to calculate the rotation between the base frame $\mathcal{R}_{b}$ and the traveling-plate frame $\mathcal{R}_{T P}$.

For the optimization of the Gough platform, we assume that positioning errors $\Delta \boldsymbol{x}_{H}$ and the length measurement errors $\Delta l$ are small enough to write an approximation of the error model such as :

$$
\Delta \boldsymbol{x}_{H} \approx \boldsymbol{J}_{H}\left(\mathcal{P}, \boldsymbol{x}_{H}\right) \Delta \boldsymbol{l}
$$

where $\mathcal{P}^{T}=\left[\begin{array}{llll}r_{b} & r_{T P} & \alpha_{b} & \alpha_{T P}\end{array}\right]^{3}$ is the vector of the geometrical parameters and $\boldsymbol{J}_{H}\left(\mathcal{P}, \boldsymbol{x}_{H}\right)$ is the Jacobian matrix of the Gough platform. Only the inverse of the Jacobian matrix has an analytical form which can be calculated as follows:

$$
\boldsymbol{J}_{\boldsymbol{H}}^{-1}=\left[\begin{array}{cc}
\boldsymbol{u}_{1} & -\boldsymbol{u}_{1} \wedge \boldsymbol{B}_{1} \boldsymbol{E}_{H} \\
\vdots & \vdots \\
\boldsymbol{u}_{6} & -\boldsymbol{u}_{6} \wedge \boldsymbol{B}_{6} \boldsymbol{E}_{H}
\end{array}\right]
$$

with

$$
\boldsymbol{u}_{i}=\frac{\boldsymbol{A}_{i} \boldsymbol{B}_{i}}{l_{i}}
$$

Fig. 2. Delta geometrical parameters 


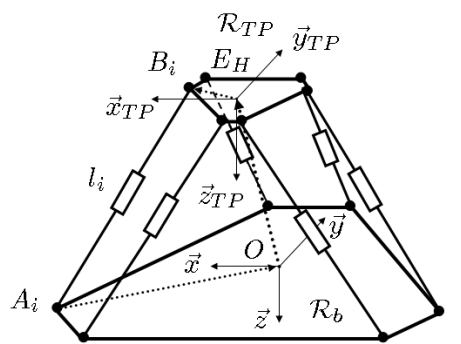

(a) 3D view

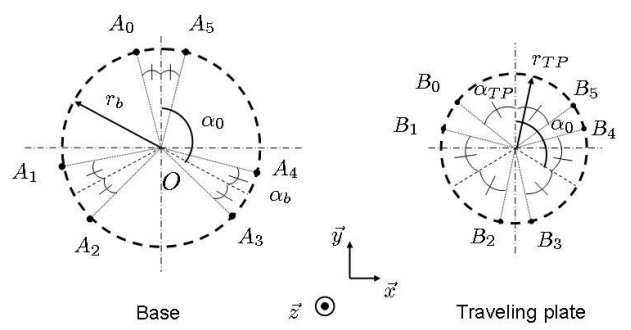

(b) Top view

Fig. 3. Geometrical parameters of the Gough platform

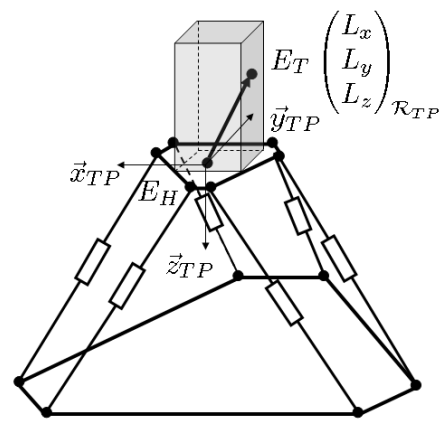

Fig. 4. Tool point $E_{T}$ and its bounding box

\section{Modeling of the Tool Point}

The optimization is performed to obtain the best resolution for the measuring system evaluated at the tool level. But the shape and the size of the tool is unknown since it can be varied depending on the machining task. The lever arm between the hexapod traveling-plate center $E_{H}$ and the tool point $E_{T}$ is variable and is defined by the vector in the base frame $\mathcal{R}_{T P}$ :

$$
\boldsymbol{E}_{H} \boldsymbol{E}_{T}=\left[\begin{array}{l}
L_{x} \\
L_{y} \\
L_{z}
\end{array}\right]_{\mathcal{R}_{T P}}
$$

In the case of this machine, as Delta mechanism impose the orientation, the vector $\boldsymbol{E}_{H} \boldsymbol{E}_{T}$ can be considered the same in the base frame and in the traveling plate frame of the Gough platform. The coordinates $L_{x}, L_{y}, L_{z}$ are bounded and the bounding box is presented in Fig. 4.

\section{E. Machine Workspace}

We designed a MT which allows to machine only small or medium-sized parts. The considered workspace $\mathcal{W}$ is a cube whose sides are 300 millimeters (Fig. 5). The optimization

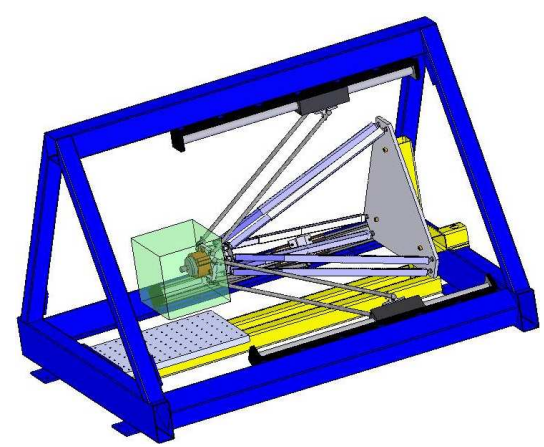

Fig. 5. MT workspace $\mathcal{W}\left(300 \times 300 \times 300 \mathrm{~mm}^{3}\right)$

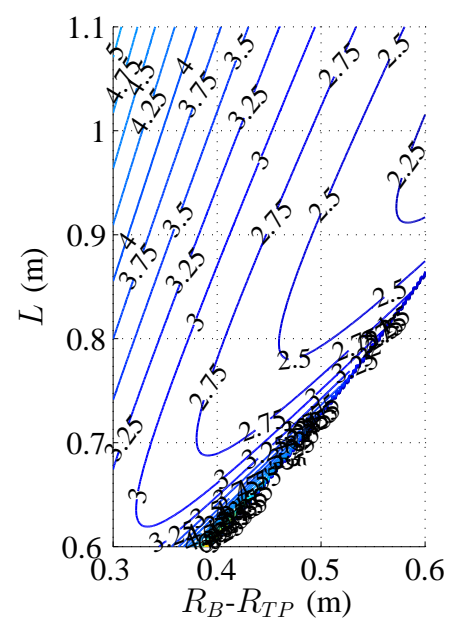

Fig. 6. Maximum of the condition number of the Jacobian matrix in a $0.3 \times 0.3 \times 0.3 \mathrm{~m}^{3}$ workspace

of the robot Delta and the Gough platform is performed in this workspace.

\section{OPTIMIZATION OF THE DELTA MECHANISM}

\section{A. Optimization Criterion}

The main quality sought for the Delta mechanism is the isotropy of its Jacobian matrix. That is to insure a homogeneous behavior in velocity and to have an accurate control of the manipulator [18].

\section{B. Optimized Delta parameters}

A grid of points is defined within the workspace $\mathcal{W}$, and for each point of this grid, the condition number of the Jacobian matrix $\boldsymbol{J}_{D}$ is calculated for variable values of $L$ and $\Delta R$. Figure 6 shows the variation of the maximum condition number according to $L$ and $\Delta R$. This figure allows to choose the parameter $\Delta R$ considering that the arms must not be too long in order to avoid too deform because of the cutting forces. So, the length of the arms are fixed to $800 \mathrm{~mm}$ and $\Delta R$ is equal to $480 \mathrm{~mm}$. The choice of these parameters is a compromise between the performances and the size of the machine. Concerning the parameter $d$, it is chosen as big as possible to decrease the orientation error of the end-effector [26]. The final values of Delta parameters are presented in Table I. 
TABLE I

OPTIMIZED DELTA PARAMETERS

\begin{tabular}{|c|c|c|c|}
\hline$R_{B}$ & $R_{T P}$ & $L$ & $d$ \\
\hline $540 \mathrm{~mm}$ & $60 \mathrm{~mm}$ & $800 \mathrm{~mm}$ & $75 \mathrm{~mm}$ \\
\hline
\end{tabular}

\section{OPTIMIZATION OF THE GOUGH PLATFORM}

\section{A. Introduction}

The Gough platform as measuring system must have good positioning capabilities: repeatability, resolution and accuracy [7]. These capacities are dependent on several factors. Some factors can be controlled during the design process while others do not. Let's see this for each positioning capability.

First of all, repeatability is extremely dependent on the realization of the joints and on the choice of the mechanical elements. Backlashes or friction on the joints, and the errors due to the finite resolution of the active-joint encoders can decrease the repeatability.

Concerning the robot accuracy, calibration is required to eliminate the positioning errors due to manufacturing errors. On the designed Gough platform, the calibration is very simple. The measuring legs can be calibrated with an artefact one by one, while the position of the spherical joint centers on the traveling plate and the base can be measured with a coordinate measuring machine. Other sources of errors exist as compliance which can be modeled and identified [12]. In our case, the compliance of the Gough platform is neglected since no stresses are supposed to act on the elements of Gough platform (except the weight of the legs which is neglected).

Finally, the last capability is the resolution of the mechanism. The first element who gets involved in the mechanism resolution is the active-joint encoders resolution. The second element is the mechanism architecture which is determined during the design phase. So, the mechanism theoretical resolution can be improved by an optimization of the dimensions of mechanism elements.

The goal of the optimization is to improve the measurement resolution at the tool level. In other words, considering the resolution of the leg encoders, the resolution at the tool level is the minimal displacement of the tool which can be detected by the measuring device. The objective is to have a measuring device which can detect the smallest possible displacement of the tool. The optimization consists in finding the dimensions of the Gough platform which allow to reach this objective.

\section{B. Optimization criterion}

Two phases are distinguished concerning the Gough platform optimization. First of all, the optimization problem is analyzed considering the tool point as known. Then, the tool bounding box will be considered.

1) Leg Encoder Errors: Any small displacement of the Gough platform traveling plate, in position $\Delta \boldsymbol{p}_{H}$ and orientation $\Delta \boldsymbol{r}_{H}$, results in a small displacement of the considered tool point can be approximated by $\Delta \boldsymbol{p}_{T}$; this displacement is evaluated at first order as follows:

$$
\Delta \boldsymbol{p}_{T}=\Delta \boldsymbol{p}_{H}+\Delta \boldsymbol{r}_{H} \times \boldsymbol{E}_{H} \boldsymbol{E}_{T}
$$

with

$$
\Delta \boldsymbol{x}_{H}=\left[\begin{array}{c}
\Delta \boldsymbol{p}_{H} \\
\Delta \boldsymbol{r}_{H}
\end{array}\right]
$$

From (5), the relation mapping the length measurement errors $\Delta \boldsymbol{l}$ to the corresponding tool positioning errors $\Delta \boldsymbol{p}_{T}$ can be written:

$$
\Delta \boldsymbol{p}_{T}=\boldsymbol{J}_{H_{P}}\left(\mathcal{P}, \boldsymbol{x}_{H}\right) \Delta \boldsymbol{l}+\boldsymbol{J}_{H_{R}}\left(\mathcal{P}, \boldsymbol{x}_{H}\right) \Delta \boldsymbol{l} \times \boldsymbol{E}_{H} \boldsymbol{E}_{T}
$$

with

$$
\boldsymbol{J}_{H}\left(\mathcal{P}, \boldsymbol{x}_{H}\right)=\left[\begin{array}{l}
\boldsymbol{J}_{H_{P}}\left(\mathcal{P}, \boldsymbol{x}_{H}\right) \\
\boldsymbol{J}_{H_{R}}\left(\mathcal{P}, \boldsymbol{x}_{H}\right)
\end{array}\right]
$$

To simplify (11), the second term of its right member is rearranged as follows:

$$
\begin{aligned}
\boldsymbol{J}_{H_{R}} \Delta \boldsymbol{l} \times \boldsymbol{E}_{H} \boldsymbol{E}_{T} & =-\boldsymbol{E}_{H} \boldsymbol{E}_{T} \times \boldsymbol{J}_{H_{R}} \Delta \boldsymbol{l} \\
& =-\widehat{\boldsymbol{E}_{H} \boldsymbol{E}_{T}} \boldsymbol{J}_{H_{R}} \Delta \boldsymbol{l}
\end{aligned}
$$

where $\widehat{\boldsymbol{E}_{H} \boldsymbol{E}_{T}}$ represents the cross-product matrix.

A small change in the length of the six hexapod legs is mapped into a displacement for the considered tool point by the following relation:

$$
\Delta \boldsymbol{p}_{T}=\boldsymbol{J}_{T} \Delta \boldsymbol{l}
$$

where

$$
\boldsymbol{J}_{T}=\boldsymbol{J}_{H_{P}}-\widehat{\boldsymbol{E}_{H} \boldsymbol{E}_{T}} \boldsymbol{J}_{H_{R}}
$$

is a $3 \times 6$ matrix.

Looking for the 'worst case' requires to find the largest value of $\left\|\Delta \boldsymbol{p}_{T}\right\|$ (Euclidean norm of the vector $\Delta \boldsymbol{p}_{T}$ ) when each measuring leg encoder suffers from an uncertainty of $\varepsilon$ equal to their resolution:

$$
-\varepsilon<\Delta l_{i}<\varepsilon
$$

Due to the linearity of the system (14), for a given point of the workspace and for a given tool point, the maximal value of $\left\|\Delta \boldsymbol{p}_{T}\left(\mathcal{P}, \boldsymbol{X}_{H}\right)\right\|$ corresponds to the $2^{6}$ possible combinations corresponding to vectors $\Delta \boldsymbol{l}$ whose components are equal to $+\epsilon$ or $-\epsilon$.

2) Unknown Tool Size: Now, for a given $\Delta l$ belonging to the $2^{6}$ combinations, the fact that the tool point is considered inside a bounding box has to be taken into account. Equation (14) can be developed as follows:

$$
\boldsymbol{\Delta}_{T}=\left[\begin{array}{l}
\sum_{i=1}^{6} J_{1 i} \Delta l_{i}+\sum_{i=1}^{6} J_{5 i} \Delta l_{i} L_{z}-\sum_{i=1}^{6} J_{6 i} \Delta l_{i} L_{y} \\
\sum_{i=1}^{6} J_{2 i} \Delta l_{i}+\sum_{i=1}^{6} J_{6 i} \Delta l_{i} L_{x}-\sum_{i=1}^{6} J_{4 i} \Delta l_{i} L_{z} \\
\sum_{i=1}^{6} J_{3 i} \Delta l_{i}+\sum_{i=1}^{6} J_{4 i} \Delta l_{i} L_{y}-\sum_{i=1}^{6} J_{5 i} \Delta l_{i} L_{x}
\end{array}\right]
$$


where $J_{j i}$ is the element at j-th row and i-th column of $\boldsymbol{J}_{H}$. The norm of $\boldsymbol{\Delta} \boldsymbol{p}_{T}$ is given by:

$$
\begin{aligned}
& \left\|\boldsymbol{\Delta} \boldsymbol{p}_{T}\right\|=\left(\left(S_{1}+S_{5} L_{z}-S_{6} L_{y}\right)^{2}\right. \\
& \left.\quad+\left(S_{2}+S_{6} L_{x}-S_{4} L_{z}\right)^{2}+\left(S_{3}+S_{4} L_{y}-S_{5} L_{x}\right)^{2}\right)^{\frac{1}{2}}
\end{aligned}
$$

with

$$
S_{j}=\sum_{i=1}^{6} J_{j i} \delta l_{i}
$$

The squared norm is then studied as a function of $L_{x}, L_{y}$ and $L_{z}$ :

$$
f\left(L_{x}, L_{y}, L_{z}\right)=\left\|\boldsymbol{\Delta} \boldsymbol{p}_{T}\right\|^{2}
$$

Finding the maxima of function $\left\|\Delta p_{T}\right\|$ is equivalent to finding the maxima of function $\left\|\Delta p_{T}\right\|^{2}$.

Briot [17] presents the mathematical background necessary to study this function. He classifies four types of maximum (first, second, third and fourth kind) which are respectively in the whole bounding box, or on the faces, or on the edges or on the corners, of the bounding box. Finally, we must study the following functions:

$$
\begin{aligned}
& f_{1}:\left(L_{x}, L_{y}, L_{z}\right) \rightarrow f\left(L_{x}, L_{y}, L_{z}\right), \\
& f_{2}:\left(L_{y}, L_{z}\right) \rightarrow f\left(L_{x_{\min }}, L_{y}, L_{z}\right), \\
& f_{3}:\left(L_{y}, L_{z}\right) \rightarrow f\left(L_{x_{\max }}, L_{y}, L_{z}\right), \\
& f_{4}:\left(L_{x}, L_{z}\right) \rightarrow f\left(L_{x}, L_{y_{\min }}, L_{z}\right), \\
& \left.f_{5}:\left(L_{x}, L_{z}\right) \rightarrow f\left(L_{x}, L_{y_{\max }}, L_{z}\right)\right), \\
& f_{6}:\left(L_{x}, L_{y}\right) \rightarrow f\left(L_{x}, L_{y}, L_{z_{\min }}\right), \\
& f_{7}:\left(L_{x}, L_{y}\right) \rightarrow f\left(L_{x}, L_{y}, L_{z_{\max }}\right), \\
& f_{8}:\left(L_{z}\right) \rightarrow f\left(L_{x_{\min }}, L_{y_{\min }}, L_{z}\right), \\
& f_{9}:\left(L_{z}\right) \rightarrow f\left(L_{x_{\min }}, L_{y_{\max }}, L_{z}\right), \\
& f_{10}:\left(L_{z}\right) \rightarrow f\left(L_{x_{\max }}, L_{y_{\min }}, L_{z}\right), \\
& f_{11}:\left(L_{z}\right) \rightarrow f\left(L_{x_{\max }}, L_{y_{\max }}, L_{z}\right), \\
& f_{12}:\left(L_{y}\right) \rightarrow f\left(L_{x_{\min }}, L_{y}, L_{z_{\min }}\right), \\
& f_{13}:\left(L_{y}\right) \rightarrow f\left(L_{x_{\min }}, L_{y}, L_{z_{\max }}\right), \\
& f_{14}:\left(L_{y}\right) \rightarrow f\left(L_{x_{\max }}, L_{y}, L_{z_{\min }}\right), \\
& f_{15}:\left(L_{y}\right) \rightarrow f\left(L_{x_{\max }}, L_{y}, L_{z_{\max }}\right), \\
& f_{16}:\left(L_{x}\right) \rightarrow f\left(L_{x}, L_{y_{\min }}, L_{z_{\min }}\right), \\
& f_{17}:\left(L_{x}\right) \rightarrow f\left(L_{x}, L_{y_{\max }}, L_{z_{\max }}\right), \\
& f_{18}:\left(L_{x}\right) \rightarrow f\left(L_{x}, L_{y_{\min }}, L_{z_{\min }}\right), \\
& f_{19}:\left(L_{x}\right) \rightarrow f\left(L_{x}, L_{y_{\max }}, L_{z_{\max }}\right),
\end{aligned}
$$

where $L_{x_{\min }}, L_{x_{\max }}, L_{y_{\min }}, L_{y_{\max }}, L_{z_{\min }}, L_{z_{\max }}$ designate the minimal and the maximal bounds of $L_{x}, L_{y}, L_{z}$.

The first function $f_{1}$ reaches a maximum when its gradient is null and when its hessian matrix is negative definite. The system of equations which described that the gradient is null is:

$$
\left\{\begin{array}{l}
S_{6}\left(S_{2}+S_{6} L_{x}-S_{4} L_{z}\right)-S_{5}\left(S_{3}+S_{4} L_{y}-S_{5} L_{x}\right)=0 \\
-S_{6}\left(S_{1}+S_{5} L_{z}-S_{6} L_{y}\right)+S_{4}\left(S_{3}+S_{4} L_{y}-S_{5} L_{x}\right)=0 \\
S_{5}\left(S_{1}+S_{5} L_{z}-S_{6} L_{y}\right)-S_{4}\left(S_{2}+S_{6} L_{x}-S_{4} L_{z}\right)=0
\end{array}\right.
$$

The three equations of this system are not independent. This system represents the equation of a line. Now, it is necessary to study the hessian matrix to qualify the critical points of the function which belong to this line:

$$
\boldsymbol{H}\left(f_{1}\right)=\left[\begin{array}{ccc}
2 S 5^{2}+2 S 6^{2} & -2 S 5 S 4 & -2 S 6 S 4 \\
-2 S 5 S 4 & 2 S 4^{2}+2 S 6^{2} & -2 S 6 S 5 \\
-2 S 6 S 4 & -2 S 6 S 5 & 2 S 4^{2}+2 S 5^{2}
\end{array}\right]
$$

This matrix is constant whatever $L_{x}, L_{y}$ and $L_{z}$. The determinant of this matrix is null and its eigenvalues are $\sigma_{1}=0$ and $\sigma_{2}=\sigma_{3}=2 S_{6}^{4}+2 S_{5}^{2}+2 S_{4}^{2}$. The matrix $\boldsymbol{H}\left(f_{1}\right)$ is positive semi-definite and the function $f$ has no maximum of the first kind.

Then, the functions $f_{2}, \ldots, f_{19}$ are treated as the first one, that is, analyzing their gradients and hessian matrix. It is determined that there is neither maximum of the second kind nor maximum of the third kind.

Finally, only a maximum of the fourth kind exists and is on one of the eight corners of the tool bounding box.

3) Global Optimization Criterion: Let $\Omega_{L_{x}}, \Omega_{L_{y}}, \Omega_{L_{z}}$, $\Omega_{l_{0}}, \Omega_{l_{1}}, \Omega_{l_{2}}, \Omega_{l_{3}}, \Omega_{l_{4}}, \Omega_{l_{5}}$ be the spaces defined by:

$$
\begin{array}{rlrl}
\Omega_{L_{x}} & =\left\{L_{x_{\min }}, L_{x_{\max }}\right\}, & \Omega_{L_{y}} & =\left\{L_{y_{\min }}, L_{y_{\max }}\right\} \\
\Omega_{L_{z}} & =\left\{L_{z_{\min }}, L_{z_{\max }}\right\}, & \Omega_{l_{0}} & =\left\{l_{0}-\varepsilon, l_{0}+\varepsilon\right\} \\
\Omega_{l_{1}} & =\left\{l_{1}-\varepsilon, l_{1}+\varepsilon\right\}, & \Omega_{l_{2}}=\left\{l_{2}-\varepsilon, l_{2}+\varepsilon\right\} \\
\Omega_{l_{3}}=\left\{l_{3}-\varepsilon, l_{3}+\varepsilon\right\}, & \Omega_{l_{4}}=\left\{l_{4}-\varepsilon, l_{4}+\varepsilon\right\} \\
\Omega_{l_{5}}=\left\{l_{5}-\varepsilon, l_{5}+\varepsilon\right\} & &
\end{array}
$$

Let $\Lambda=\Omega_{L_{x}} \times \Omega_{L_{y}} \times \Omega_{L_{z}} \times \Omega_{l_{0}} \times \Omega_{l_{1}} \times \Omega_{l_{2}} \times \Omega_{l_{3}} \times \Omega_{l_{4}} \times \Omega_{l_{5}}$ be the cartesian product of the spaces defined above.

Finally, the optimization criterion is given by:

$$
\begin{gathered}
\mathcal{C}_{\text {opt }}=\max _{\left(x_{H}, y_{H}, z_{H}\right) \in \mathcal{W}} \mathcal{C}_{\text {int }} \\
\mathcal{C}_{\text {int }}=\max _{\lambda \in \Lambda}\left\|\Delta \boldsymbol{p}_{T}\left(\mathcal{P}, \boldsymbol{x}_{H}, \lambda\right)\right\|
\end{gathered}
$$

Then, the optimization consists in finding the vector of parameters $\mathcal{P}^{*}$ which minimize the criterion $\mathcal{C}_{\text {opt }}$.

\section{Gough platform parameters}

The Gough platform optimization has to take into account the Delta geometry to avoid collisions. The distance between the center of the two structures is chosen such as it is the smallest possible to minimize the size and the weight of the traveling plate. This distance is equal to $0.1 \mathrm{~m}$. Another parameters are not introduced in the optimization process. Indeed, the hexapod leg lengths can vary between two bounds chosen by the designers $\left(l_{i} \in[634 \mathrm{~mm}, 1080\right.$ $m m])$.

A preliminary study showed that the optimization criterion of the Gough platform is better if the angles $\alpha_{b}$ and $\alpha_{T P}$ are small and if the radius $r_{b}$ and $r_{T P}$ are big. Considering this and the collisions aspect a first set of parameters is chosen to initialize the optimization algorithm.

The final values of Gough platform are presented in Table II.

Finally, the parameter optimization allows to reach a theoretical resolution evaluated at the tool level about $20 \mu \mathrm{m}$ for a resolution of the leg encoders of $1 \mu \mathrm{m}$. 
TABLE II

GOUGH PLATFORM PARAMETERS

\begin{tabular}{|c|c|c|c|}
\hline$r_{b}$ & $r_{T P}$ & $\alpha_{b}$ & $\alpha_{T P}$ \\
\hline $375 \mathrm{~mm}$ & $75 \mathrm{~mm}$ & $6^{\circ}$ & $40^{\circ}$ \\
\hline
\end{tabular}

\section{CONCLUSION}

In this paper we have proposed the design and the optimization of a parallel machine-tool composed of (i) an actuated parallel 3-dof mechanism (a linear Delta) and (ii) a measuring 6-dof mechanism (a Gough platform). We have explained the interest for a machine-tool to have a measuring device independent of the actuated mechanism notably to measure the consequence of its deformations due to the machining stresses. Finally, we have proposed an optimization which is performed to obtain the best resolution for the measuring system evaluated at the tool level. The final design of the coming prototype is shown in Fig. 1. Different control strategies will be evaluated on the prototype for example online calibration, compensation or control of the machine in the measurement system space.

\section{ACKNOWLEDGMENTS}

This work has been partially funded by the European project NEXT, acronyms for "Next Generation of Production Systems", Project No. IP 011815.

\section{REFERENCES}

[1] J. Merlet, Les robots parallèles. Paris: Hermès, 1997.

[2] V. E. Gough, "Contribution to discussion of papers on research in automotive stability, control and tyre performance," in Proc. Auto Div., Institute of mechanical engineering, 1956-1957.

[3] P. Sheldon, "Six axis machine tool," U.S. Patent 5388935 , Feb., 1995

[4] R. Clavel, "Conception d'un Robot Parallèle Rapide à 4 Degrés de Liberté," Ph.D. dissertation, EPFL, Lausanne, Switzerland, 1991.

[5] O. Company, F. Pierrot, F. Launay, and C. Fioroni, "Modelling and preliminary design issues of a 3-axis parallel machine-tool," in PKM'2000 : International Conference on Parallel Kinematic Machines, Ann Arbor, USA, September 13-15 2000, pp. 14-23.

[6] E. Krause and Co, "Machining system with movable tool head," U.S. Patent 6161992 , Dec., 2000.

[7] B. W. Mooring, Z. S. Roth, and M. R. Driels, Fundamentals of Manipulator Calibration. New-York: John Wiley \& Sons, Inc., 1991.

[8] J. Tlusty, J. Ziegert, and S. Ridgeway, "A comparison of stiffness characteristics of serial and parallel machine tools," Journal of Manufacturing Systems, vol. 2, no. 2, pp. 67-76, 2000.

[9] F. Marquet, "Contribution à l'Étude de l'Apport de la Redondance en Robotique Parallèle," Ph.D. dissertation, Université Montpellier II, Montpellier, France, 2002.
[10] C. M. Gosselin and D. Zang, "Stiffness analysis of parallel mechanisms using a lumped model," International Journal of Robotics and Automation, vol. 17, no. 1, pp. 17-27, 2002.

[11] G. Ecorchard and P. Maurine, "Self-calibration of delta parallel robots with elastic deformation compensation," in Proc. IEEE International Conference on Intelligent Robots and Systems (IROS'05), Edmonton, Alberta, Canada, August 2005.

[12] D. Deblaise, X. Hernot, and P. Maurine, "A systematic analytical method for pkm stiffness matrix calculation," in IEEE International Conference on Robotics and Automation (ICRA'06), Orlando, Floride, USA, May 2006.

[13] P. Renaud, N. Andreff, F. Pierrot, and P. Martinet, "Combining endeffector and legs observation for kinematic calibration of parallel mechanisms," in IEEE International Conference on Robotics and Automation (ICRA'04), News Orleans, USA, April 26-May 12004, pp. 4116-4121.

[14] T. Arai, R. Stoughton, K. Homma, H. Adachi, T. Nakamura, and K. Nakashima, "Development of a parallel link manipulator," in IEEE International Conference on Advanced Robotics (ICAR'91), Pisa, Italy, June 1991, pp. 839-844.

[15] J.-P. Merlet, "Computing the worst case accuracy of a PKM over a worskpace or a trajectory," in Proc. Parallel Kinematics Seminar (PKS'06), Chemnitz, Germany, April 2006.

[16] J. Merlet, "Jacobian, manipulability, condition number, and accuracy of parallel robots," ASME Journal of Mechanical Design, vol. 128, pp. 199-205, January 2006.

[17] S. Briot and I. Bonev, "Accuracy analysis of 3DOF planar parallel robots," Mech. Mach. Theory, doi:10.1016/j.mechmachtheory.2007.04.002.

[18] C. Gosselin and J. Angeles, "A global performance index for the kinematic optimization of robotics manipulators," International Journal of Mechanical Design, vol. 113, no. 3, pp. 220-226, 1991.

[19] A. Yu, I. Bonev, and P. Zsombor-Murray, "Geometric approach to the accuracy analysis of a class of 3-DOF planar parallel robots," Mech. Mach. Theory, doi:10.1016/j.mechmachtheory.2007.03.002.

[20] R. Katz, Z. Li, and F. Pierrot, "Conceptual design of a high speed drilling machine (HSDM) based on PKM module," in Technical Report, ERC/RMS, The University of Michigan, 2001.

[21] L. W. Tsai and S. Joshi, "Kinematics and optimization of a spatial 3-UPU parallel manipulator," ASME Journal of Mechanical Design vol. 122, p. 439446, December 2000.

[22] J. M. Hervé and F. Sparacino, "Star, a new concept in robotics," in International Symposium on Robotics, Mecatronics and Manufacturing Systems (IMACS'92), vol. 1, September 1992, p. 176183.

[23] C. Reboulet, C. Lambet, and N. Nombrail, "A parallel redundant manipulator: Speed-R-Man and its control," in International Symposium on Robotics And Manufacturing (ISRAM'92), November 11-13 1992 , p. 285291.

[24] D. Chablat, P. Wenger, and J. Angeles, "Conception isotropique dune morphologie parallèle : application l'usinage," in International Conference On Integrated Design and Manufacturing in Mechanical Engineering (IDMME'00), Montral, Canada, May 2000.

[25] O. Company, "Machines-outils Rapides à Structure Parallèle. Méthodologie de Conception, Applications et Nouveaux Concepts,' Ph.D. dissertation, Université Montpellier II, Montpellier, France, 2000

[26] P. Vischer, "Improve the Accuracy of Parallel Robot," Ph.D. dissertation, École polytechnique fédérale de Lausanne, Lausanne, Switzerland, 1996. 